\title{
Analysis of Scheduling the Independent CCHBs for Partially Reconfigurable FPGA
}

\author{
Bharathi N. ${ }^{1 *}$ and P. Neelamegam² \\ 'School of Computing, SASTRA University, Thanjavur 613401, India; bharathi_n@cse.sastra.edu \\ ${ }^{2}$ School of Electrical \& Electronics Engineering, SASTRA University, \\ Thanjavur613401, India; Neelkeer@yahoo.com
}

\begin{abstract}
The emerging reconfigurable computing reduces the need of computation exhaustive applications, which are always demanding more efficient computation hardware. The partially reconfigurable Field Programmable Gate Arrays (FPGA) are highly suitable for performance improvement. This paper discusses the study of FPGA utilization when scheduling fixed size configurable computation hardware block (CCHB) by applying a heuristic. Based on the parameters (speedup, CCHB size etc.,) associated with the independent CCHBs, scheduling is performed and it is repeated for various sizes of FPGA. From the study of four applications from benchmark suite, it is observed that the device utilization is increased with size of CCHBs not greater than 0.5 times or not less than 0.85 times of the size of FPGA.
\end{abstract}

Keywords: Field Programmable Gate Array (FPGA), Scheduling, Configurable Computation Hardware Block (CCHB), Response Time, Utilization.

\section{Introduction}

Field programmable gate array has made a revolution in VLSI technology and it made the hardware programming flexible. It is one of the reconfigurable hardware, which is suitable for high speed demanding applications like multimedia and computation intensive applications like cryptography [10] and image processing and control system [9] applications. FPGA provides facility of configure by the application developer and also it supports partial reconfiguration [3] that is similar to rewriting the information in memory.

In this work the FPGA is represented as 2 Dimensional logic cells. The configurable computation hardware block (CCHB) is the computation intensive part of the application that can be executed in one instruction cycle if it is configured in the reconfiguration hardware. CCHBs are taken from four (Adpcm Dec., Adpcm Enc., Blowfish and Sha) benchmark applications from Mibench benchmark suite $[5,7]$ occupies certain number of logic cells in the FPGA to perform its operation. More than one CCHB can be configured based on the logic cell availability in the FPGA. If the FPGA cannot accommodate all the CCHBs, then after the execution completion by currently configured CCHBs, the waiting CCHBs is reconfigured [13]. Here, the issue is which CCHB should be configured for the first round and which one in the second round. To reduce the response time of $\mathrm{CCHB}$, maintaining the order among them with the use of scheduling is desirable.

Traditional perception of scheduling is a process of allocating task for execution in the processing unit based on any single factor. On the advent of field programmable gate array, the scheduling process is highly improved as a new way of allocating task either in the CPU or in the FPGA [14] by considering several factors, which are not even considered, in the traditional counterpart. Scheduling can be classified

* Corresponding author:

Bharathi N. (bharathi_n@cse.sastra.edu) 
as offline scheduling, on line scheduling $[17,8]$, preemptive scheduling, non-preemptive scheduling etc. In addition to the existing classification, based on the type of the code, the flavors are the software tasks, the hardware tasks or CCHBs and hybrid tasks. The scheduling can be performed either statically or dynamically. The dynamic scheduling $[12,6]$ allocates the resource for the arriving task as soon as they arrived provided enough resource is available; otherwise the task will be inserted into the waiting queue where tasks are queued based on the gain. The static scheduler stores the set of arrived tasks in a pool. The set of tasks are sorted based on their gain periodically. The sorted tasks are then scheduled and resource is allocated based on the availability of the resource.

In the previous works, the researchers deal with maximum two parameters associated with the tasks, which are periodic in nature. Zhou et al. [17] discusses the scheduling based on various queues and modules separately. But the queues overhead and its management is additional burden. It is eliminated in this work by considering single pool of tasks. Yi Lu et al. [8] focuses on application completion time and single task response time. In a study [1] placement during scheduling is discussed without considering the relocation delay. Yin et al. [16] considered the real time tasks with deadline. Steiger et al. [14] motivated the OS frames and evaluates performance and runtime efficiency. Belviranli et al. [2] focused on self scheduling scheme in heterogeneous microprocessors. The work of Liu et al. [11] deals with hardware/software partitioning and then scheduling. The previous works are not concentrated on the utilization of the reconfigurable hardware. Hence this paper focuses mainly the utilization of reconfigurable hardware, influence of CCHB size on utilization etc.
The first part of this paper explains the architecture framework to schedule the CCHB based on pre-scheduler module. Second, the working of the system for effectively scheduling the CCHB based on the gain factors calculated in the pre-scheduler module. Finally the results are discussed based on the graphs plotted against various parameters and the observed performance improvement.

\section{The Architecture Framework}

The architecture consists of the pre-scheduler, scheduler, configuration manager, reconfigurable device interface and the FPGA. The CCHB blocks are collected from the hardware-software partitioning module $[7,15]$ and stored in a pool as shown in Figure 1. Parameters which are associated with each CCHB block such as speedup, CCHB size, CPU cycles needed if executed in software are provided to the pre-scheduler. The scheduler block determines the order in which the CCHBs are configured in the FPGA to facilitate improving the utilization of device. The configuration manager collects the configuration bits of CCHBs for each round and reconfigures the device through the interface.

The pre-scheduler collects the parameters associated with CCHB blocks, which are to be considered in the scheduling process. Gain factor is derived from the parameters collected. Based on gain factor, the task configuration order is determined. Each parameter contributes to gain factor in one of 2 ways. Speedup is the ratio between the amounts of time required to execute the task in software to that of hardware. So it will improve the gain factor. Similarly the other parameters either improve or weaken the gain factor. Though the pre-scheduler can be a module inside the scheduler or it can be a module in the application itself,

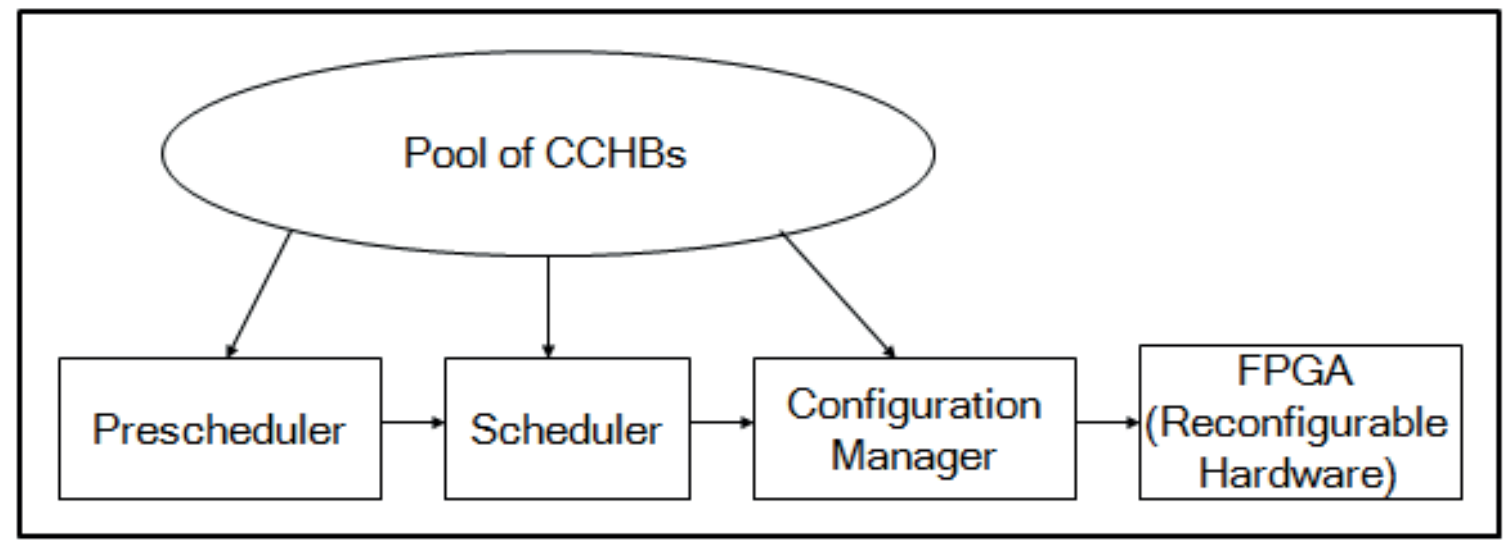

Figure 1. Architecture of the scheduling process. 
implementing it as a separate unit is advisable. In order to provide uniformity in calculating the gain factor, separate pre-scheduler module is better than keeping in the application software.

The scheduler collects the gain factors computed for each CCHBs and its associated data needed for scheduling from the pre-scheduler. The scheduler sorts the CCHBs based on the gain factor and schedules for FPGA. During scheduling it checks whether the relocation needed or not. Configuration relocation is the concept of moving an already configured CCHB in order to accommodate the incoming CCHB. After scheduling process, the CCHBs are identified, which are configured currently in the FPGA and their movement to accommodate the incoming CCHB is decided. The above case is possible only when the collection of free logic cells is greater than or equal to the new incoming $\mathrm{CCHB}$. Then for each gain factor the relocation delay [4] is also included. Gain factor of a CCHB may be high if relocation delay is not considered, at the same time if relocation is considered the gain factor may goes down for the same CCHB.

The configuration manager gathers the information about order of CCHBs from Scheduler. It also accesses pool of CCHBs to transfer the configuration bits of scheduled CCHBs to the FPGA through device interface. The configuration manager can also be considered as PostScheduler, which is responsible for the configuration. The scheduler may reject some of the CCHBs because of their poor contribution to the performance improvement. These CCHBs are filtered in the scheduling phase itself. They are not taken into account in the post-scheduling phase. The FPGA hardware is considered as a $2 \mathrm{D}$ array as in existing works [1]. CCHBs are also specified as $2 \mathrm{D}$ array. The CCHBs are executed in the FPGA till their completion. Afterwards other waiting CCHBs are configured by reconfiguration.

\section{Implementation}

CCHB parameters are the input to the pre-scheduler module. It will calculate the gain based on the equation 1 . The calculated gain values are sorted in decreasing order. $\mathrm{CCHB}$ that is giving high gain is at the first in the sorted list. The scheduler checks the free logic cells availability for each CCHB and allocates it in the hardware if logic cells available. The input given to the scheduler is in terms of its size, its execution time and if needed deadline along with the gain factor. The gain factors taken into account are speedup, size of task, frequency of occurrence, CPU cycles consumption in software (if same speedup), relocation delay such as time spent in deciding which $\mathrm{CCHB}$ to relocate, $\mathrm{CCHB}$ relocating delay, etc.,(if it requires), deadline (in Real time tasks), etc.

$$
\text { F(GF1, GF2, GF3, ..., GFn) }
$$

and the relocation delay factor for each gain factor if needed should be calculated as

$$
\begin{gathered}
\mathrm{GFj}=\mathrm{F}(\mathrm{RDF} 1, \mathrm{RDF} 2, \ldots, \mathrm{RDFm}) \\
\text { Where } 1<\mathrm{j}<\mathrm{n}
\end{gathered}
$$

The gain factors can be chosen based on the application(s), which are to be configured on the FPGA. In this work, the gain is taken as

(Speedup ratio * CPU cycles consumption (if executed as software)) / CCHB size.

The FPGA size is also given as input to the scheduler module. Based on the size of FPGA the CCHBs are allocated. The hardware cycle time are almost equal to the software cycle time excluding of instruction fetch and decoding stages in software. The same instructions can be occurred several times and that is included in the task gain. Hardware blocks of the repeated occurrences are not reconfigured and $\mathrm{CCHB}$ size is one of the main factors in reducing the number of configurations and contributing largely for FPGA utilization. CCHB size is also considered in the task gain, and scheduling is performed by considering the order of occurrence if the CCHBs are dependent on another.

\section{Results and Discussion}

\subsection{Simulation Setup}

The simulation work is programmed in $\mathrm{C}$ under windows in Intel Core2Duo processor with $1.60 \mathrm{GHz}$. The CCHB sizes considered are 576, 864, $1296 \& 2304$ logic cells. The FPGA sizes assumed are 3136, $4096 \& 5184$ logic cells. The sizes of both FPGA [18] and CCHB are in terms of logic cells/configurable logic blocks.

The achieved scheduling of CCHB is based on the gain factors estimated in the pre-scheduler unit. First all the CCHB sizes are kept fixed (E.g. Any one size from above mentioned) and for FPGA of size 3136 logic cells, scheduling is examined and the response time and device utilization are observed. Next, the CCHB sizes are taken as variable (any size from above mentioned - mixed) and scheduling is repeated for same FPGA sizes taken and the 
utilization and response time is recorded. The graph is plotted for Device utilization and response time against CCHB size with the FPGA size as fixed. This is repeated for various FPGA sizes.

The possible CCHB sizes for the applications are taken and scheduled with different $\mathrm{CCHB}$ sizes. The Table 1 shows the required cells for each application and the optimum CCHB size within the considered set of CCHB sizes. The data in first 4 rows is when the applications are executed in separate configurations. If the applications are combined with possible combinations and executed then the requirement is different and its corresponding optimum $\mathrm{CCHB}$ size is shown in Table 1.

\subsection{The Effect of CCHB Size}

It is observed from the simulation that the CCHB size is highly influencing the scheduling response time and also the utilization of the hardware. The CCHB sizes taken for this are $24 \times 36,24 \times 24,36 \times 36,24 \times 24$ and $36 \times 36$, $36 \times 48,48 \times 48$ and the mixture of the specified sizes (mixed).

The size of CCHB should be less than $50 \%$ or greater than $85 \%$ of the hardware size in order to utilize the hardware efficiently. Otherwise the utilization of hardware is poor. As shown in figure 2 the utilization is high for task set of size $36 \times 36(1296-0.32$ times $)$ which is less than 0.5 times of the FPGA size and low for task set of size $48 \times 48$

Table 1. Application requirement and its optimum CCHB sizes

\begin{tabular}{lccc}
\hline Application & No. of basic operations & Required no. of cells & Optimum CCHB size (cells) \\
\hline Blowfish & 640 & 1280 & $36 \times 36$ \\
Sha & 122 & 244 & $24 \times 24$ \\
Adpcm_Dec & 18 & 36 & $24 \times 24$ \\
Adpcm_Enc & 17 & 34 & $24 \times 24$ \\
Blowfish, Sha & 762 & 1524 & $36 \times 48$ \\
Blowfish, Adpcm_Dec & 658 & 1316 & $36 \times 48$ \\
Blowfish, Adpcm_Enc & 657 & 1314 & $36 \times 48$ \\
Sha, Adpcm_Dec, Adpcm_Enc & 157 & 314 & $24 \times 24$ \\
Blowfish, Sha, Adpcm_Dec, Adpcm_Enc & 797 & 1594 & $36 \times 48$ \\
\hline
\end{tabular}

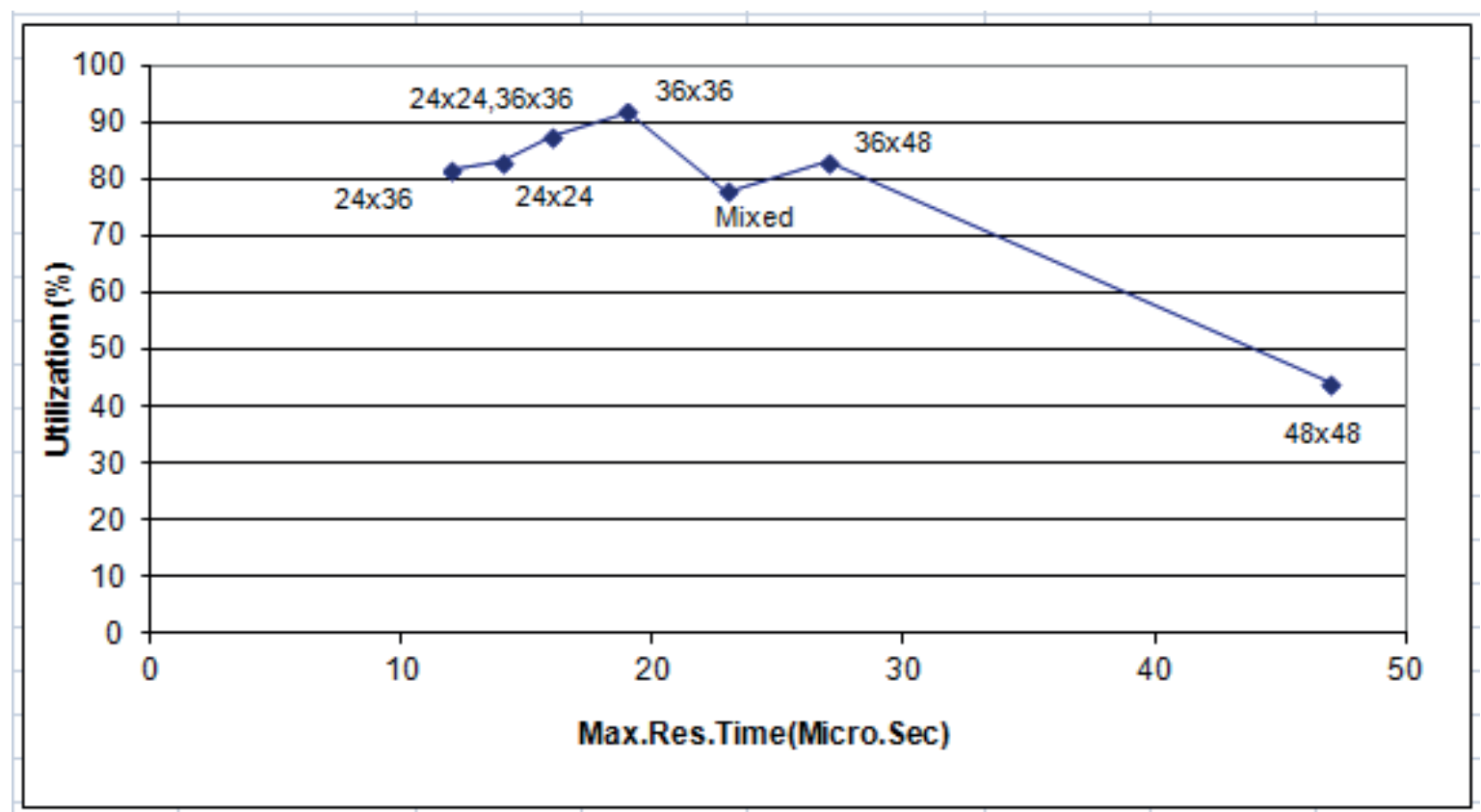

Figure 2. Max. Response time and Utilization for various CCHB sizes scheduled in FGPA of size 4096 logic cells. 
(2304 - 0.56 times) which is greater than 0.5 times and less than 0.85 times of the FPGA size taken.

\subsection{The Effect of FPGA Size}

The influence of FPGA size is shown in Figure 3 and Figure 4 , it is observed, that the response time decreases as the size of FPGA increases.

\subsection{The Effect of CCHB Execution Time}

The CCHB execution time also influences response time directly and hence the utilization indirectly. As the $\mathrm{CCHB}$ execution time increased, obviously the scheduling response time increased. Utilization is affected for the mixed size of CCHB case as shown in utilization graph of
Figure 3 and Figure 4. If the execution time of the largest $\mathrm{CCHB}$ is increased then utilization is decreased with the largest $\mathrm{CCHB}$ just occupying $75 \%$ of hardware. This is observed only in mixed $\mathrm{CCHB}$ size case. Utilization is affected constantly during scheduling for the other cases. Figure 5 shows the software cycles saved percent for each application as actual performance improvement.

\section{Conclusion}

The gain factors taken for scheduling, increases the performance of the computing system. It is observed that the CCHB size selection is highly influencing the response time and utilization of the reconfigurable hardware. The proposed scheduling process is used for the mixed $\mathrm{CCHB}$
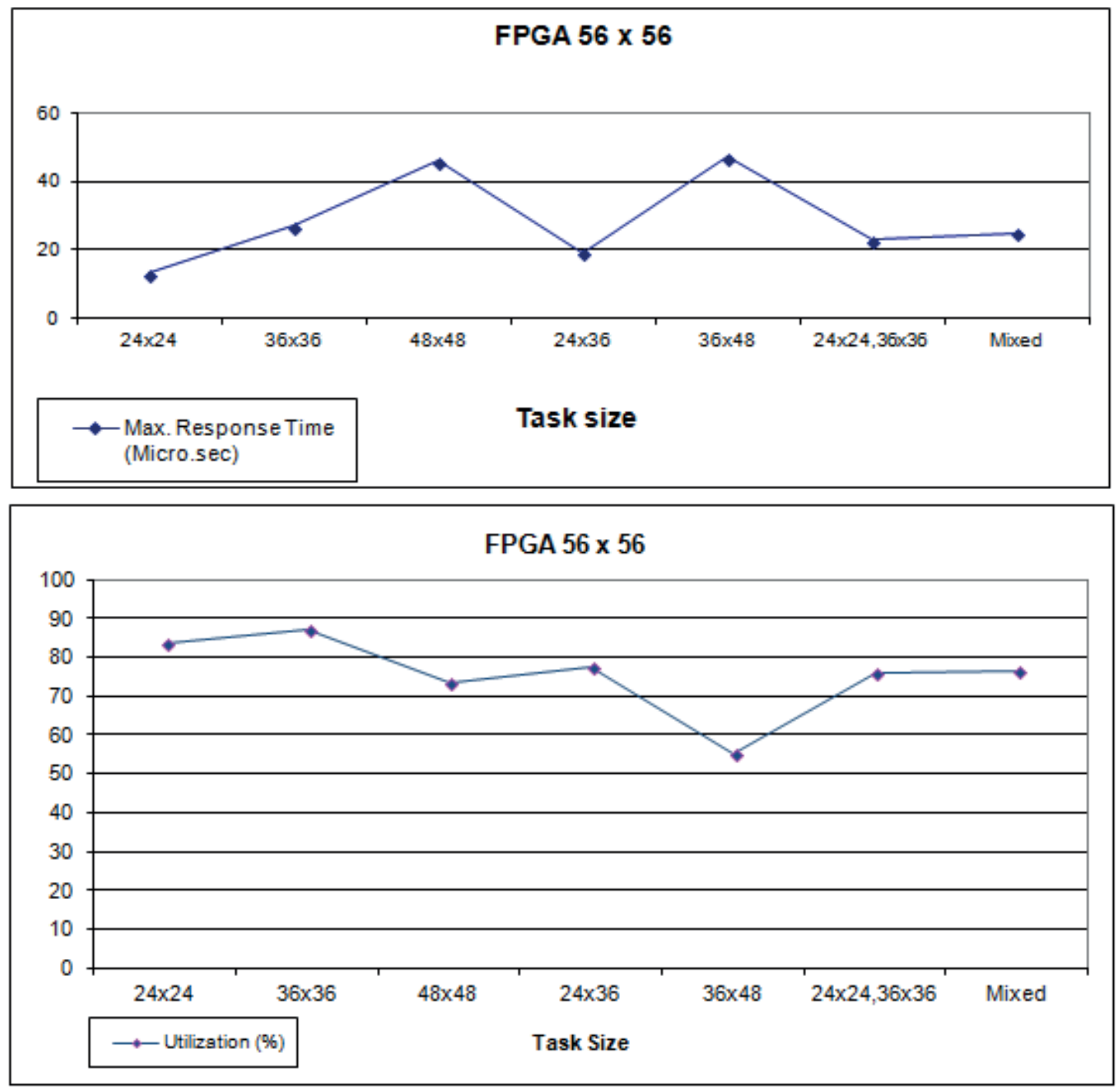

Figure 3. Max. Response time and Utilization for various CCHBs scheduled in FPGA of size 3136 logic cells. 

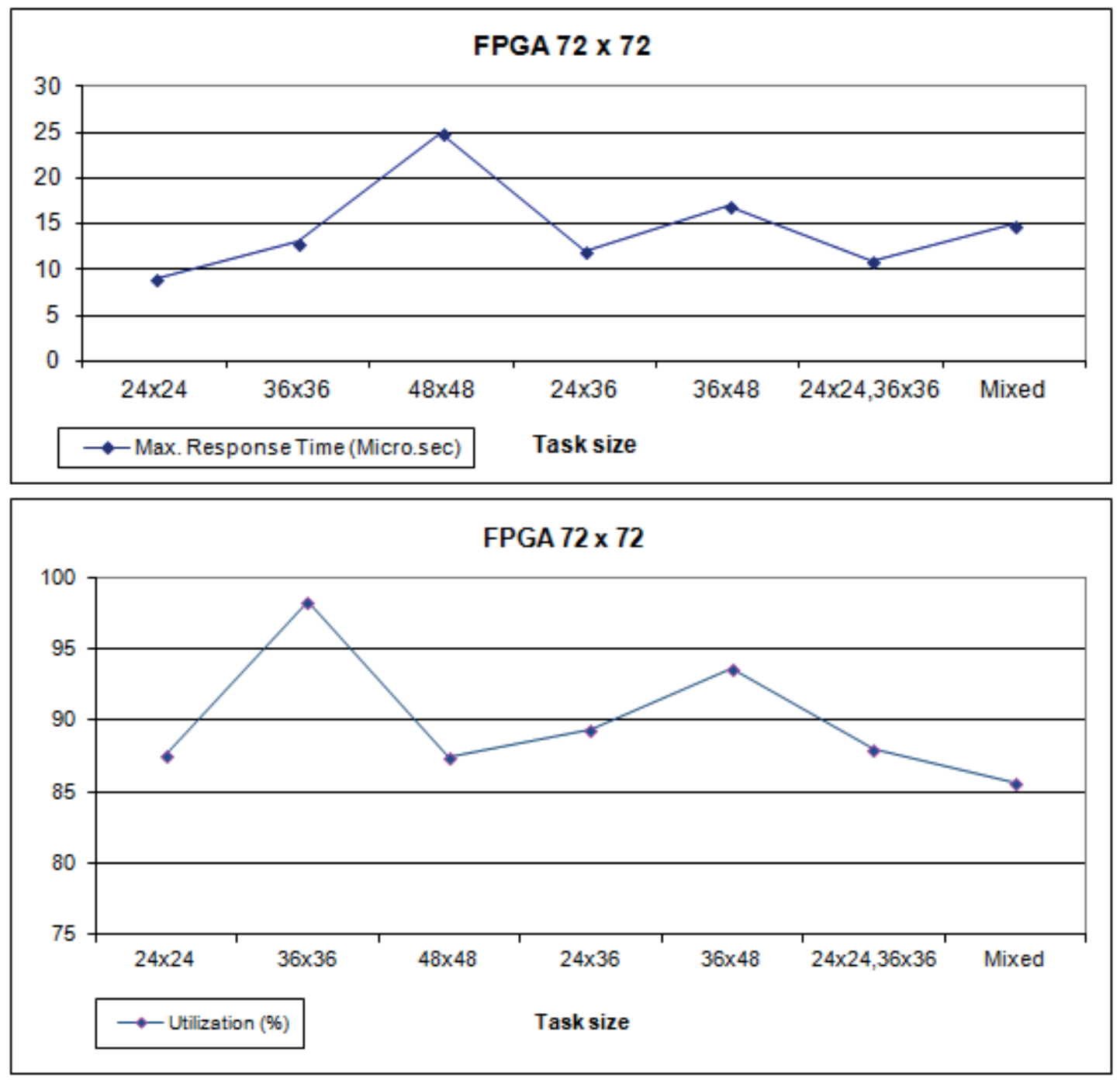

Figure 4. Max. Response time and Utilization for various CCHBs scheduled in FPGA of size 5184 logic cells.

size and also for various FPGA sizes. From this work, it is concluded that the CCHB size should be less than 0.5 times and greater than 0.85 times of the reconfigurable hardware to get better response time and utilization. It is also observed that the mixed CCHB case shows higher utilization and lesser response time as FPGA size increases, with the same set of CCHB.

\section{References}

1. Ahmadinia A, Bobda C et al. (2004). Task scheduling for heterogeneous reconfigurable computers, Proceedings of the 17th Symposium on Integrated Circuits and System Design, 22-27.

2. Belviranli M E, Bhuyan L N et al. (2013). A dynamic selfscheduling scheme for heterogeneous multiprocessor architectures, Transactions on Architecture and Code Optimization, vol 9(4).

3. Carvalho E, Calazans $\mathrm{N}$ et al. (2004). PADReH - A framework for the design and implementation of dynamically and partially reconfigurable systems. Proceedings of the 17th Symposium on Integrated Circuits and System Design, 10-15.

4. Compton K, Li Z et al. (2002). Configuration relocation and defragmentation for run-time reconfigurable computing, IEEE Transactions on Very Large Scale Integration (VLSI) Systems, vol 10(3), 209-220.

5. Guthaus M R, Ringenberg J S et al. (2001). MiBench: A free, commercially representative embedded benchmark suite, IEEE International Workshop on Workload Characterization WWC-4, 3-14.

6. Hong C, Benkrid K et al. (2011). Efficient on-chip task scheduler and allocator for reconfigurable operating systems, IEEE Embedded Systems Letters, vol 3(3), 85-88. 


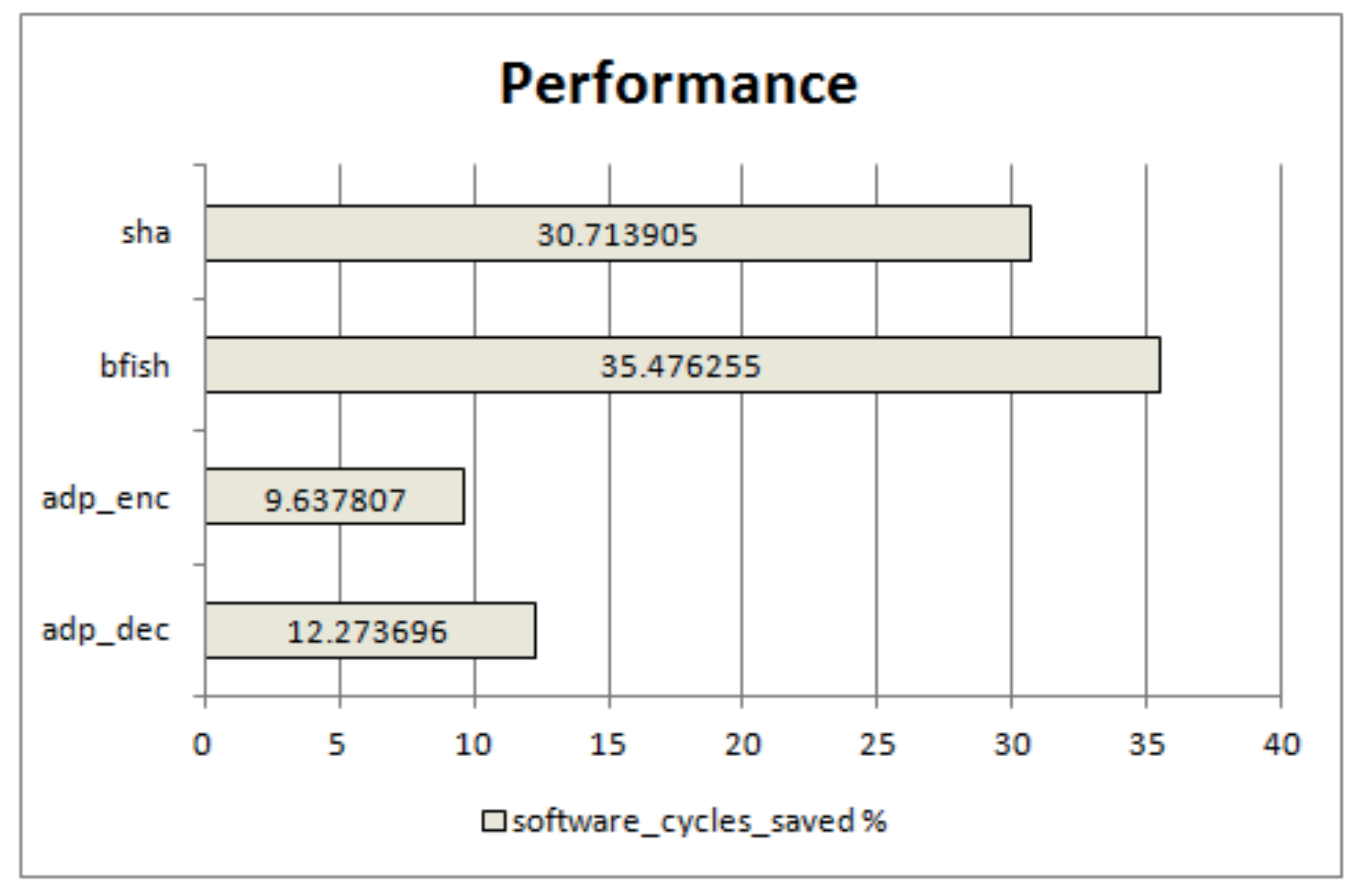

Figure 5. Software cycles saved percentage for each application.

7. Lam S K, Bharathi N K et al. (2006). Efficient management of custom instructions for run-time reconfigurable instruction set processors, IEEE International Conference on Field Programmable Technology, 261-264.

8. Lu Y, Marconi T et al. (2009). Online task scheduling for the FPGA-based partially reconfigurable systems, Proceedings of the 5th International Workshop on Reconfigurable Computing: Architectures, Tools and Applications, vol (5453), 216-230.

9. Martín P, Bueno E et al. (2012). An FPGA-based approach to the automatic generation of VHDL code for industrial control systems applications: A case study of MSOGIs implementation, Mathematics and Computers in Simulation, In Press, Corrected Proof, Available online 27 July 2012.

10. Sokouti M, Sokouti B et al. (2010). FPGA implementation of improved version of the Vigenere cipher, Indian Journal of Science and Technology, vol 3(4), 459-462.

11. Liu P, Wu J et al. (2012). Hybrid algorithms for hardware/software partitioning and scheduling on reconfigurable devices, Mathematical and Computer Modelling, In Press, Corrected Proof, Available online 16 November 2012.

12. Resano J, Mozos D et al. (2005). A Hybrid Prefetch Scheduling Heuristic to Minimize at Run-Time the Reconfiguration Overhead of Dynamically Reconfigurable Hardware,
Proceedings of the Conference on Design, Automation and Test in Europe, vol 1, 106-111.

13. Bhandari S U, Subbaraman S et al. (2010). Internal dynamic partial reconfiguration for real time signal processing on FPGA, Indian Journal of Science and Technology, vol 3(4), 365-368.

14. Steiger C, Walder $\mathrm{H}$ et al. (2004). Operating systems for reconfigurable embedded platforms: online scheduling of real-time tasks, IEEE Transactions on Computers, vol 53(11), 1393-1407.

15. Todman T J, Constantinides G A et al. (2005). Reconfigurable computing: architectures and design methods, IEEE conference Proceedings on Computers and Digital Techniques, vol 152(2), 193-207.

16. Yin J, Zheng B et al. (2012). A hybrid real-time fault-tolerant scheduling algorithm for partial reconfigurable system, Journal of Computers, vol 7(11), 2773-2780.

17. Zhou X, Liang L et al. (2008) Online Task Scheduling for Heterogeneous Reconfigurable Systems, 11th International Conference on Computer Supported Cooperative Work in Design IV, vol 5236, 596-607.

18. Xilinx Platform FPGAs. [Online]. Available from: http:// www.xilinx.com 